\title{
Improved Brain Extraction Tool using Marker-Controlled Watershed Segmentation
}

\author{
Kalavathi P. \\ Department of Computer Science and Applications Gandhigram Rural Institute - Deemed University, Tamil \\ Nadu, India.
}

\begin{abstract}
MRI head scan images contains some non-brain tissues and it is necessary to remove these nonbrain tissues for further processing. The Brain Extraction Tool (BET) is one of the most popular brain segmentation technique which removes the non-brain tissues in MRI head scan volumes. It is a simple and robust brain extraction method. It is a boundary based method and uses deformable surface to detect brain boundaries. Due to its over smoothing capabilities it always under-segment the brain images by including more non-brain tissues in its final segmentation. The aim of this proposed method is to overcome the problems encountered with the existing BET method by using the marker-controlled watershed segmentation. The proposed improved brain extraction tool (IMBET) was tested with 20 volumes of brain images and its performance was compared with the popular existing methods BET, BSE, WAT, HWA and GCUT. The computed similarity measures Dice $(D)$ and Jaccard $(J)$ show that the proposed method outperform the BET and produced better brain extraction result than the existing methods.
\end{abstract}

Keywords : Brain extraction tool (BET), improved BET, skull stripping, brain segmentation, MRI segmentation, brain extraction

\section{INTRODUCTION}

Medical imaging modality is a set of techniques which are used to create images of the human body and plays an inevitable role in early detection, diagnosis, therapy and monitoring of disease/disorder by providing detailed information on patient's anatomy. Among the various medical imaging techniques, MRI is the most widely used imaging technique in the medical field. MRI is particularly suitable for brain imaging. MR images of the brain and other cranial structures are clearer and more detailed than the other imaging methods. These features make MRI an invaluable tool in early diagnosis and evaluation of many brain related deceases. Brain image segmentation plays a significant role in numerous applications in biomedical imaging. A number of techniques have been proposed to segment the brain from MRI head scan images [1]-[21]. Brain Extraction Tool (BET)[1], Brain Surface Extraction (BSE)[2], Watershed Algorithm (WAT)[3], Hybrid Watershed Algorithm (HWA)[4] and Skull stripping using Graph Cuts (GCUT)[5] are the popular methods. The various imperfections introduced in MRI head scan images such as low resolution, high level of noise, low contrast, geometric deformations and presence of imaging artifacts [22] are the overhead for the development of efficient brain segmentation algorithm.

In this paper, the existing method BET is improved based on marker-controlled watershed technique. This proposed method (IMBET) is quantitatively and qualitatively evaluated on 20 volumes of brain images obtained from Internet Brain Segmentation Repository (IBSR)[23]. The performance of the proposed method was compared with the existing methods BET, BSE, WAT, HWA and GCUT. The remaining part of the paper is organized as follows: Section II presents the description about the BET and IMBET methods. The results and discussion are given in Section III and the conclusion is given in Section IV.

\subsection{Brain Extraction Tool}

II. METHOD

BET is an automated Brain Extraction Tool [1] designed to segment the brain and non-brain tissues in MRI head scan volumes. It is a boundary based method and uses deformable model which evolvescontour to fit the brain's surface based on its computed energy forces. The initial contour of BET is initialized with a tessellated mesh and pushes the mesh to the brain boundary based on the calculated smoothing forces and pushing forces. These energy forces are computed by applying a set of locally adaptive model forces. BET tool is freely available in the internet in MRIcro 1.4 version and it comprises the following major steps to extract the brain from MRI head scan images.

Steps involved in BET method:

- Histogram based threshold estimation

- Binarization of input image based on the estimated threshold

- Finding the center of gravity of the head image 
- Initializing the triangular tessellated sphere surface

- $\quad$ Surface deformation based on the computed energy forces

- Extraction of brain boundary

Although, BET is a robust and fast brain extraction tool it has some drawbacks. The main drawback of BET method is often it over smooth the brain image due to the global pushing force and thus it under-segment the brain image by including several non-brain tissues in its final segmentation [24]. As a result of this, many of the existing brain extraction methods such as BSE, WAT, HWA and GCUT outperform the BET. Therefore in this proposed method, the output of the BET result is improved by applying the marker-controlled watershed segmentation technique.

\subsection{Watershed Segmentation}

The watershed transform [25] [26] is a popular and most widely used segmentation method in the field of image processing especially in medical image processing. Watershed is considered to be a topographic region growing method. Watershed transform concept is based on visualizing an image in three dimensions; i.e., two spatial coordinates versus gray levels. For topographic interpretation three points were considered; (i) Points belonging to a regional minimum (ii) Points at which a drop of water would fall with certainty to a single minimum and (iii) Points at which water would be equally likely to fall to more than one such minimum. The objective of this method is to find the watershed lines from these concepts.

There are mainly three methods to implement watershed; Distance Transform Approach, Gradient method and Marker-Controlled Approach. In distance transform approach, the distance measure functions are used as watersheds transform function to segment the image. The gradient method [27] pre-process the gray scale image before applying the watershed transform. Usually the gradient is high at the border of the object and low inside the object. The gradient based method produce over-segmentation by introducing more contours. The over-segmentation problem due to the direct application of gradient prior to watershed transform is solved by marker-controlled watershed approach [28]. The markers are used to modify the gradient image. Usually, the markers are placed inside an object of interest in order to reduce the unwanted internal contour in the watershed transformation.

\subsection{Brain Extraction by Improved Watershed Segmentation (IMBET)}

In this proposed method, the marker-controlled watershed transformation is applied to improve the brain extraction result of BET method. Segmentation by marker controlled watershed works better than the gradient method. The input brain image is first applied on BET method, the output of BET is used as an input for this proposed method. The brain extraction process of the proposed method is illustrated in Fig. 1. Image (b) of Fig. 1 is the resultant skull stripped image of Figure 1(a) by BET. The binary image is obtained using the method medical image binarization using square wave representation given in [29]. Since the BET often under-segment the image and it has introduced many non-brain tissues in its final segmentation, therefore it is necessary to disconnect these non-brain tissues. For this purpose the morphological erosion and dilation operations are used. Then the proposed method selects the largest connected component (LCC) in this image because it is found that the brain region in the binary image is LCC. The binary image and the selected LCC mask are shown in Fig. 1(c) and Fig. 1(d) respectively. Then the LCC image is obtained using the LCC mask as per the following equation.

$$
L C C=\left\{\begin{array}{ll}
f(x, y) & \text { if } \operatorname{LCCmask}(x, y)=1 \\
0 & \text { otherwise }
\end{array} .\right.
$$

where, $f(x, y)$ is the BET output image. Then the gradient magnitude of the LCC image is obtained to detect the edges. There are different operators are used for gradient through convolution such as Sobel, Prewitte, Robert, Canny etc., Edge detection operator are implemented with convolution masks which are often discrete approximations to differential operators. In this method Sobel convolution mask is used to obtain the gradient image. Sobel mask is a discrete differentiation operator, computing an approximation of the opposite of the gradient of the image intensity function. The Sobel operator is based on convolving the image with a small, separable, and integer valued filter in horizontal and vertical direction and is therefore relatively inexpensive in terms of computations. The Sobel operator uses two $3 \times 3$ kernels which are convolved with the original image to calculate approximations of the horizontal $G_{x}$ and vertical $G_{y}$ derivatives. If $f$ is the source image, then the computation of $G_{x}$ and $G_{y}$ are as follows: 


$$
G_{x}=\left[\begin{array}{lll}
-1 & 0 & +1 \\
-2 & 0 & +2 \\
-1 & 0 & +1
\end{array}\right] * f \text { and } G_{y}=\left[\begin{array}{rrr}
+1 & +2 & +1 \\
0 & 0 & 0 \\
-1 & -2 & -1
\end{array}\right] * f
$$

where * denotes the 2-dimensional convolution operation.

The $x$-coordinate is defined here as increasing in the "right"-direction, and the $y$-coordinate is defined as increasing in the "down"-direction. At each point in the image, the resulting gradient approximations can be combined to get the gradient magnitude as given below:

$$
G=\sqrt{G_{x}^{2}+G_{Y}^{2}}
$$

In order to reduce the noise present in the gradient image (Fig. 1(e)), the anisotropic diffusion filter[30] is applied. This technique aimed to reduce the image noise without affecting the edges, lines and other image features. The diffused image for the sample selected image is shown in Fig. 1(f). It is used as a markercontroller for watershed transformation. The watershed segmentation is performed on the diffused image to get the final segmentation. The result of watershed transform and the skull stripped brain images are given in Fig. $1(\mathrm{~g})$ and Fig. 1(h) respectively. The summary of the steps involved in this proposed method is given in Algorithm 1.
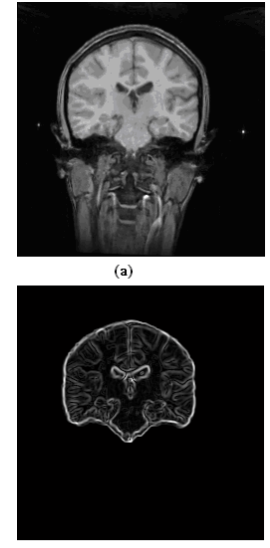
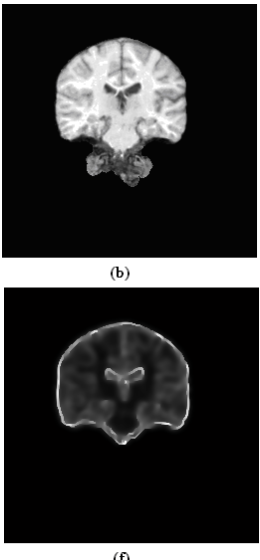

(f)

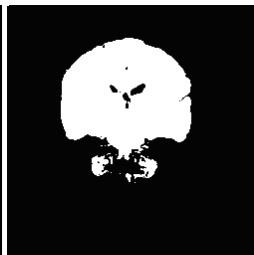

(c)

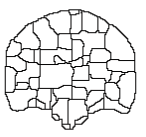

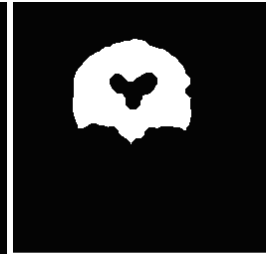

(d)

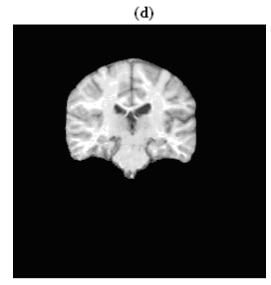

(h)

Fig. 1: Process of brain extraction by the proposed method; (a) Original image (b) Brain extraction by BET

(c) Binary image (d) LCC mask image (e) Gradient image (f) Diffused image (g) Result of watershed segmentation (h) Extracted brain by the proposed IMBET method

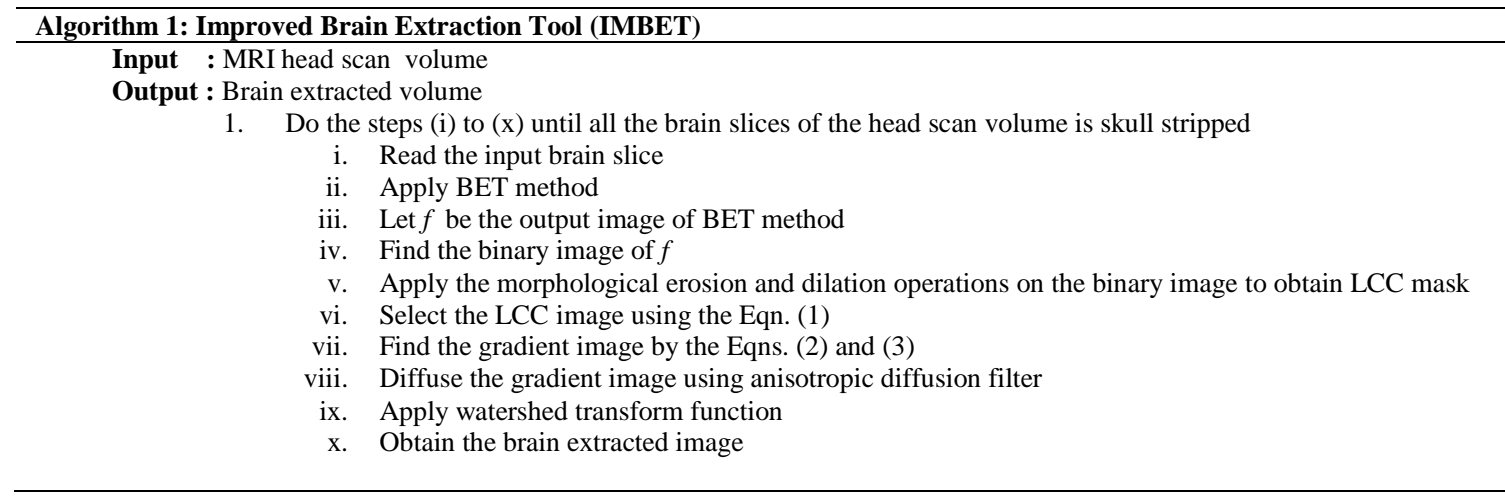

\section{RESULTS AND DISCUSSION}

This proposed method was tested with twenty volumes of T1-weighted images obtained from IBSR [24] of the Centre for Morphometric Analysis (CMA) at the Massachusetts General Hospital. It contains MR brain volumes obtained from young-middle aged normal individuals. Each volume consists of T1-weighted 2D sequential coronal slices with the dimensions of 256x256 pixels. The number of slices ranges from 60 to 65 and the slice thickness is $3.1 \mathrm{~mm}$.

To evaluate the performance of the proposed method, Jaccard $(J)$ and Dice $(D)$ similarity measures [20] were calculated. The Jaccard $(J)$ and Dice $(D)$ measures are given by: 


$$
J\left(S_{1}, S_{2}\right)=\frac{\left|S_{1} \cap S_{2}\right|}{\left|S_{1} \cup S_{2}\right|}
$$

The Dice coefficient $(D)[34]$ is given by:

$$
D\left(S_{1}, S_{2}\right)=\frac{2\left|S_{1} \cap S_{2}\right|}{\left|S_{1}\right|+\left|S_{2}\right|}
$$

where, $S_{1}$ represents the total pixels of the image obtained by the proposed method and $S_{2}$ represents the total pixels in the image obtained from ground truth data (gold standard).

The parameter setting for the existing method BET and the proposed method IMBET are given in Table 1. Both BET and IMBET were used the values as suggested by Hartly et al., [31]. The default parameter values and the values used by BET and IMBET are given in the same table.

Table 1: Parameter setting for the BET and the proposed IMBET method

\begin{tabular}{|l|l|l|l|}
\hline Method & Parameter & Default value & Value used \\
\hline \multirow{3}{*}{ BET and } & Diffusion Iteration & 3 & 3 \\
IMBET & Diffusion Constant & 25 & 35 \\
& Edge Constant & 0.64 & 0.62 \\
& Erosion Size & 1 & 2 \\
\hline
\end{tabular}

The computed $D$ and $J$ values for the selected 20 volumes of brain images are given in Table 2. This proposed method has produced better and consistent performance on all the 20 volumes of brain images. Some of the selected sample images along with the result of BET and the proposed IMBET are shown in Fig. 2. Column (a) of Fig. 2 is an original image, the output of BET is given in Fig. 2(b) and the result obtained by the proposed IMBET is shown in Fig. 2(c). For all these selected images the proposed IMBET have produced better and consistent result and thus it refined the output of the existing BET method.

The results of the proposed method are also compared with the existing BET as well as other well known existing methods BSE, WAT, HWA and GCUT. The computed mean, standard deviation and the range for both similarity measures Dice $(D)$ and Jaccard $(J)$ on the selected dataset is given in Table 3. Compared to the existing methods, the proposed IMBET method have produced better $D$ and $J$ measure. The minimum and maximum range of $D$ and $J$ value on the selected dataset are also given in Table 3. It is evident from Fig. 2, Table 2 and Table 3 that the proposed method produced better and consistent performance on the selected dataset compared to the existing methods BET, BSE, WAT, HWA and GCUT. The over-segmentation is the major problem in BET and it was prevailed in the IMBET method.

\begin{tabular}{|c|c|c|c|}
\hline S.No & Volume Name & Dice $(D)$ & $\begin{array}{l}\text { Jaccard } \\
(\mathrm{J})\end{array}$ \\
\hline 1 & 1_24 & 0.83 & 0.75 \\
\hline 2 & 2_4 & 0.87 & 0.82 \\
\hline 3 & $4 \_8$ & 0.87 & 0.82 \\
\hline 4 & $5 \_8$ & 0.77 & 0.72 \\
\hline 5 & 6_10 & 0.75 & 0.68 \\
\hline 6 & 7_8 & 0.81 & 0.75 \\
\hline 7 & 8_4 & 0.84 & 0.81 \\
\hline 8 & $11 \_3$ & 0.87 & 0.82 \\
\hline 9 & $12 \_3$ & 0.86 & 0.81 \\
\hline 10 & $13 \_3$ & 0.88 & 0.87 \\
\hline 11 & $15 \_3$ & 0.87 & 0.83 \\
\hline 12 & $16 \_3$ & 0.86 & 0.81 \\
\hline 13 & $17 \_3$ & 0.83 & 0.77 \\
\hline 14 & $100 \_23$ & 0.92 & 0.88 \\
\hline 15 & $110 \_3$ & 0.84 & 0.80 \\
\hline 16 & $111 \_2$ & 0.84 & 0.83 \\
\hline 17 & $112 \_2$ & 0.87 & 0.82 \\
\hline 18 & 191_3 & 0.84 & 0.79 \\
\hline 19 & $202 \_3$ & 0.89 & 0.85 \\
\hline \multirow[t]{2}{*}{20} & 205_3 & 0.90 & 0.86 \\
\hline & Mean & 0.85 & 0.80 \\
\hline
\end{tabular}

Table 2: Computed values of $D$ and $J$ by the proposed IMBET method for the selected dataset. 
Improved Brain Extraction Tool using Marker Controlled Watershed Segmentation

Table 3: Computed values of mean, standard deviation and range for $D$ and $J$ for BET, BSE, WAT, HWA and GCUT along with the proposed IMBET method

\begin{tabular}{|c|c|c|c|c|c|c|c|}
\hline \multicolumn{2}{|c|}{ Measures / Methods } & BET & BSE & WAT & HWA & GCUT & $\begin{array}{c}\text { IMBET } \\
\text { (proposed) }\end{array}$ \\
\hline \multirow{3}{*}{$\begin{array}{l}\text { Dice } \\
\text { (D) }\end{array}$} & Mean & 0.74 & 0.79 & 0.76 & 0.78 & 0.85 & 0.85 \\
\hline & STD & 0.14 & 0.21 & 0.14 & 0.21 & 0.09 & 0.04 \\
\hline & Range & $0.53-0.90$ & $0.00-0.95$ & $0.47-0.92$ & $0.16-0.88$ & $0.49-0.90$ & $0.75-0.92$ \\
\hline \multirow{3}{*}{$\begin{array}{c}\text { Jaccard } \\
\text { (J) }\end{array}$} & Mean & 0.61 & 0.69 & 0.64 & 0.68 & 0.75 & 0.80 \\
\hline & STD & 0.18 & 0.22 & 0.21 & 0.21 & 0.10 & 0.05 \\
\hline & Range & $0.36-0.81$ & $0.00-0.90$ & $0.09-0.78$ & $0.09-0.78$ & $0.33-0.81$ & $0.68-0.88$ \\
\hline
\end{tabular}
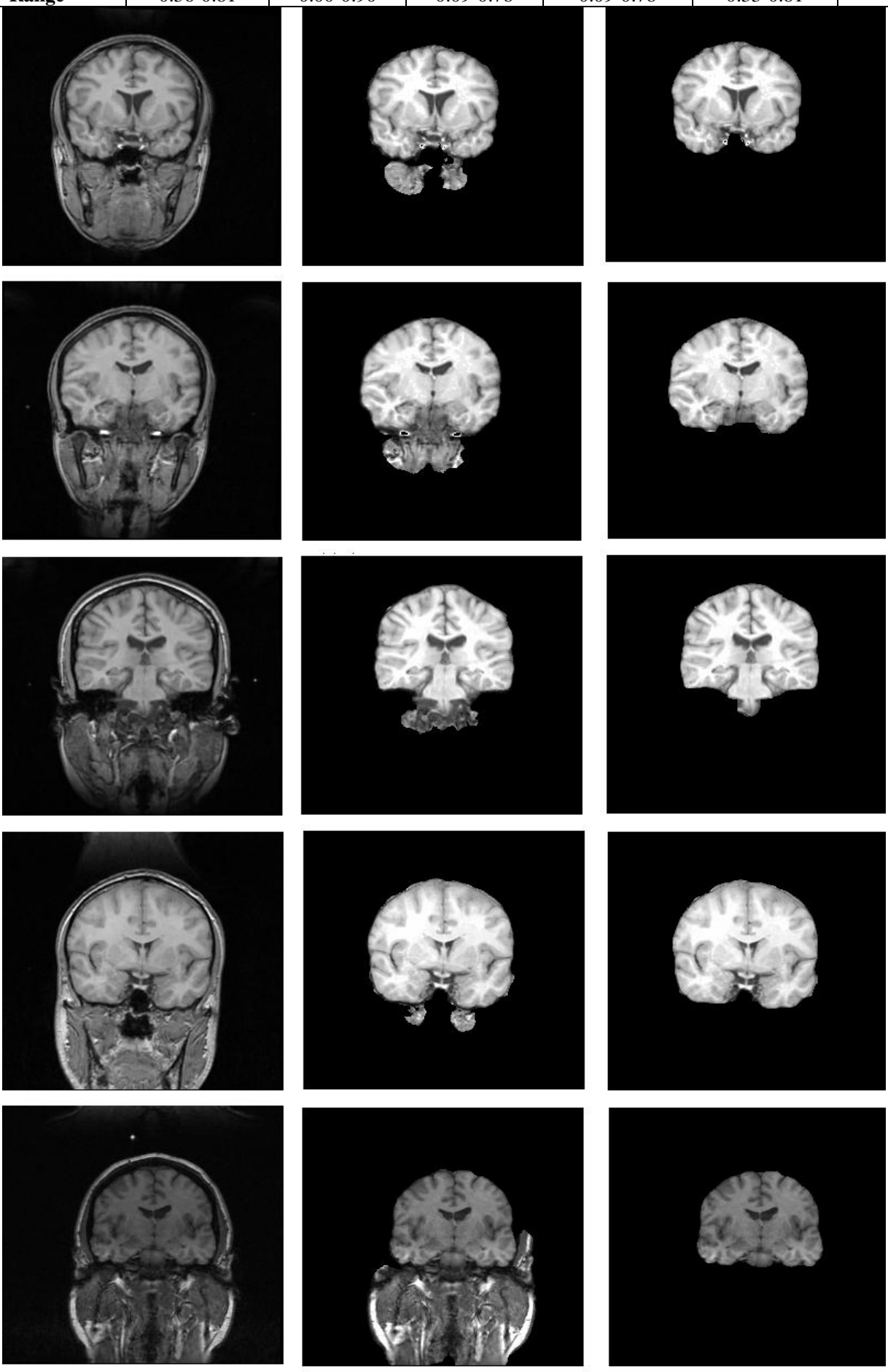

(a)

(b)

(c)

Fig. 2: Brain extraction result by the proposed IMBET method; (a) Original image (b) Brain extraction by BET (c) Brain extraction by the proposed IMBET method 
IV.

CONCLUSION

The proposed IMBET method is designed to eliminate the drawbacks of the popular brain extraction tool (BET). This proposed method has been devised using marker-controlled watershed method and it was tested with 20 volumes of brain images. The computed Dice (D) and Jaccard (J) values by the proposed method was compared with the existing method and found to produce better result. The brain is accurately segmented in all the images of the selected brain volume and thus this proposed IMBET method may play a vital role in biomedical imaging applications.

\section{REFERENCES}

[1] Smith SM., Fast Robust Automated Brain Extraction, Human Brain Mapping, 17(3), 2002, 143-155.

[2] Shattuck DW, Sandor-Leahy SR, Schaper KA, Rottenberg DA, Leahy RM., Magnetic Resonance Image Tissue Classification using a Partial Volume Model, Neuroimage, 13(5), 2001, 856-876.

[3] Hahn HK, Peitgen HO., The Skull Stripping Problem in MRI Solved by Single 3D Watershed Transform, Proc. of Medical Image Computing and Computer Assisted Intervention (MICCAI), LNCS, 1935, 2000, 134-143.

[4] Segonne F, Dale AM, Busa E, Glessner M, Salat D, Hahn HK, Fischl B., A Hybrid Approach to the Skull Stripping Problem in MRI, Neuroimage, 22, 2004, 1060-1075.

[5] Sadananthan AS, Zheng W, Chee WL, Zagorodnov V., Skull Stripping using Graph Cuts, Neuroimage,49, 2010, 225-239.

[6] Somasundaram, K. and Kalavathi. P., Automatic Skull Stripping of Magnetic Resonance Images (MRI) of Human Head Scans using Image Contour, (Image Processing, Allied Publisher, New Delhi, 2010), 147-151.

[7] Somasundaram, K. and Kalavathi. P., A Hybrid Method for Automatic Skull Stripping of Magnetic Resonance Images (MRI) of Human Head Scans, IEEE Xplore Digital Library, 2010, 1-5.

[8] Zhuang AH, Valentino DJ, Toga AW. Skull Stripping Magnetic Resonance Images using a Model-based Level Sets, NeuroImage, 32, 2006, 79-92.

[9] Park GJ, Lee C. Skull Stripping Based on Region Growing for Magnetic Resonance Images, Neuroimage, 47, 2009, 1394-1407.

[10] Carass A, Cuzzocreo J, Wheeler MB, Bazin PL, Resnick SM. Prince JL., Simple Paradigm for Extra-Cerebral Tissue Removal: Algorithm and Analysis, NeuroImage. 56(4), 2011, 1982-1992.

[11] Zhang H, Liu J, Zhu Z. Li H., An Automated and Simple Method for Brain MR Image Extraction, BioMedical Engineering OnLine, 10(81), 2011.

[12] Leung KK, Barnes J, Modat M, Ridgway GR, Bartlett JW, Fox NC, Ourselin S., Brain MAPS: An Automated, Accurate and Robust Brain Extraction Technique using a Template Library, NeuroImage, 55(3), 2011, 1091-1108.

[13] Hwang J, Han Y, Park H. Skull-Stripping Method for Brain MRI using a 3D Level Set with a Speedup Operator, Journal of Magnetic Resonance Imaging, 34(2), 2011, 445-456.

[14] Somasundaram, K. and Kalavathi. P., Skull Stripping of MRI Head Scans Based on 2D Region Growing, Proc. of ICOM'11, Anna University of Technology, Tiruchirappalli, Tamil Nadu, India, 2011, 18-23.

[15] Somasundaram, K. and Kalavathi. P., Skull Stripping of MRI Head Scans based on Chan-Vese Active Contour Model, International Journal of Knowledge Management \& e-learning, 3(1), 2011, 7-14.

[16] Somasundaram, K. and Kalavathi. P., A Novel Skull Stripping Technique for T1-weighted MRI Human Head Scans, ACM Digital Library, 2012, 1-8.

[17] Kalavathi. P., Brain Tissue Segmentation in MR Brain Images using Otsu's Multiple Thresholding Technique, IEEE Xplore Digital Library, 2013, 638-642.

[18] Eskildsen SF, Coupe P, Fonov V, Manjón JV, Leung KK, Guizard N, Wassef SN, Ostergaard LR, Collins DL., BEaST: Brain Extraction Based on Non-Local Segmentation Technique, NeuroImage, 59(3), 2012, 2362-2373.

[19] Galdames FJ, Jaillet F, Perez CA, An Accurate Skull Stripping Method Based on Simplex Meshes and Histogram Analysis in Magnetic Resonance Images, Journal of Neuroscience Methods, 206(2), 2012, 109-113.

[20] Somasundaram, K. and Kalavathi. P., Contour-Based Brain Segmentation Method for Magnetic Resonance Imaging Human Head Scans, Journal of Computer Assisted Tomography, 37(3), 2013, 353-368.

[21] Somasundaram, K. and Kalavathi. P., Brain Segmentation in Magnetic Resonance Human Head Scans using Multi-Seeded Region Growing, Imaging Science Journal, 62(5), 2014, 273-284.

[22] Somasundaram, K. and Kalavathi. P., Analysis of Imaging Artifacts in MR Brain Images, Oriental Journal of Computer Science \& Technology, 5(1), 2012, 135-141.

[23] IBSR dataset. Available Online: http://www.cma.mgh.havard.edu/ibsr/index. html

[24] Jiang S., Zhang W., Wang Y. and Chen Z.,, Brain extraction from cerebral MRI volume using a hybrid level set based active contour neighborhood model, BioMedical Engineering OnLine, 2013, 12:31.

[25] Gonzalez RC, Woods RE. Digital Image Processing, (Addison-Wesley Publishing Company. 1992)

[26] Haris, K., Hybrid image segmentation using watersheds and fast region merging, IEEE Trans Image Processing, 7(12), 1998, 1684-1699.

[27] Jackway PT. Gradient watersheds in morphological scale space, IEEE Trans Image Proc, 5, 1999, 913-921.

[28] Chi Zhang, Ning Zhang, Chengjun Li, Guoping Wang, Marker-Controlled Perception-Based Mesh Segmentation, Third International Conference on Image and Graphics (ICIG'04), 2004, 390-393.

[29] Somasundram K, Kalavathi P. Medical Image Binarization using Square Wave Representation, CCIS, Springer, 140, 2011,151-158.

[30] Perona P and Malik J, Scale-space and edge detection using anisotropic diffusion, IEEE Transactions on Pattern Analysis and Machine Intelligence, 12(7), 1990, 629-639.

[31] Hartley SM, Scher AL, Kort ESC, White LR, Launer LJ., Analysis and Validation of Automated Skull Stripping Tools: A Validation Study based on 296 MR Images from Honolulu Asia Aging Study, Neuroimage, 30, 2006, 1179-1186. 